\title{
Sedation During Neurocritical Care
}

\author{
Nieves Vanaclocha ${ }^{1}$ Vicente Chisbert ${ }^{2}$ Vicent Quilis ${ }^{3,4,5} \quad$ Federico Bilotta $^{6} \quad$ Rafael Badenes $^{7}$
}

${ }^{1}$ Department of Plastic Reconstructive Surgery, Hospital

Universitario y Politécnico La Fe, Valencia, Spain

${ }^{2}$ Escuela de Doctorado, Universidad Católica de Valencia,

"San Vicente Mártir," Spain

${ }^{3}$ Department of Neurosurgery, Hospital Clínic Universitari de

València, Spain

${ }^{4}$ College of Medicine and Science, Mayo Clinic, United States

${ }^{5}$ Department of Human Anatomy and Embryology, Faculty of

Medicine, University of Valencia, Spain

6Department of Anesthesiology, University of Rome "La Sapienza,"

Rome, Italy

${ }^{7}$ Department of Anesthesiology and Surgical-Trauma Intensive Care, Hospital Clinic Universitari Valencia, University of Valencia, Spain
Address for correspondence Rafael Badenes, MD, PhD, Department of Anesthesiology and Surgical-Trauma Intensive Care, Hospital Clinic Universitari Valencia, University of Valencia, Spain (e-mail: rafaelbadenes@gmail.com).

J Neuroanaesthesiol Crit Care 2019;6:56-61
Abstract
Keywords
- sedative agents
- neurocritical care
- neurointensive care unit
- neurological wakeup test

Sedation is an essential therapeutic strategy in the care of neurocritical patients. Intravenous sedative agents are the most widely used, with promising alternatives (dexmedetomidine, ketamine, and volatile agents) to propofol and midazolam arising. Studies designed to evaluate superiority and avoid biases are required. A neurological awakening test is safe in most patients. Potential risks and benefits of limiting deep sedation and daily interruption of sedation in these patients remain unclear. The aim of this review was to report recent clinical evidence on sedation in this subgroup of patients, focusing on its effects on clinical prognosis.

\section{Introduction}

Sedation in the neurointensive care unit (NICU) is a key element of the therapeutic strategy. ${ }^{1}$ In patients with acute brain injury (ABI), it not only limits the stress response to critical illness, provides anxiolysis, and improves patient-ventilator synchrony, but it also helps control intracranial pressure (ICP), reduces the cerebral metabolic rate, and suppresses seizures. ${ }^{2,3}$

In the general intensive care unit (ICU), recent emphasis on early weaning from mechanical ventilation and reducing ventilator-associated pneumonia has forwarded the introduction of guidelines focusing on reducing mechanical ventilation duration and ICU length of stay (LOS), while ensuring comfort and preventing further neurological damage. ${ }^{4}$ In this respect, the American Society of Critical Care Medicine published recently a summary on the use of sedatives in the ICU. ${ }^{5}$ Light over deep sedation and non-benzodiazepine sedatives over benzodiazepines were recommended, but sedation in patients with ABI was not specifically addressed.

\section{received}

February 4, 2019

accepted

March 18, 2019

published online

June 6, 2019
In the NICU, the cerebral effects of sedation have encouraged its use as a primary rather than adjuvant form of treatment. ${ }^{6}$ In fact, a study that extracted and combined data from two large, randomized, pharmacologic clinical trials on tirilazad in patients with traumatic brain injury (TBI) found that over $90 \%$ of them had received sedation. ${ }^{7}$ However, little information is available on analgesia, sedation, and delirium in the NICU, and publications addressing outcomes and pharmacologic treatment responses lack consistency and/or scientific rigor. ${ }^{8}$ Hence, clinical practice varies depending on institutional and national protocols, as well as on individual patients' response to sedation, which differs between patients and over time. It is also this lack of evidence that prevents the generation of established recommendations on sedation specifically addressed to patients with $\mathrm{ABI}$.

The aim of this review was to report the available clinical evidence regarding sedation in neurocritical care setting.

Copyright $\odot 2019$ Indian Society of Neuroanaesthesiology and Critical Care

\section{License terms \\ ()(1) $\odot \circledast$}

$10.1055 / \mathrm{s}-0039-1688897$

ISSN 2348-0548. 


\section{Intravenous Sedative Agents}

No single intravenous sedative agent has shown superiority over others, an improvement in prognosis, or a decrease in mortality. Few studies include washout periods between the administration of different sedative agents, making them susceptible to confounding effects and hampering the independent evaluation of each agent.

Propofol is the most commonly used intravenous sedative agent. ${ }^{9}$ Other agents are frequently compared with it to assess their applicability in the NICU and potential benefits over propofol. It helps keep ICP low and does not affect cerebral microdialysate composition. ${ }^{10-12}$ Propofol-induced hypotension; however, is not rare. ${ }^{10}$ The neuroprotective properties attributed to it in animal and invitro studies have not been confirmed in clinical trials. ${ }^{10,13}$ Propofol is one of the preferred intravenous sedative agents. Its short duration of action ensures a relatively rapid cessation of its effects after interrupting its infusion. Propofol-based sedation allows to accomplish neurological wakeup test (NWT) and weaning from mechanical ventilation in shorter time span than benzodiazepines-based sedation. ${ }^{14}$ These features, added to its good ICP control, make it the recommended sedative agent in the latest TBI management Brain Trauma Foundation guidelines. ${ }^{15}$ However, despite multiple efforts, its putative neuroprotective properties remain unproven. ${ }^{10,12,13}$ Propofol-related infusion syndrome (PRIS) is a rare but often lethal complication of the commonly used propofol. It is mostly associated with high doses and long-term infusion of propofol (90\% of the described cases in the literature were ICU patients). The syndrome was first described in children who received the drug in high doses over a prolonged time (average infusion rates, $7.5-10 \mathrm{mg} / \mathrm{kg} / \mathrm{h}$; total infusion time, 66-115 hours), and guidelines were subsequently adapted with restrictions in the application of propofol in children. ${ }^{16}$ Later reports described the occurrence of PRIS also in adults and demonstrated an enormous variety of clinical presentations. ${ }^{17}$ It was observed both in patients receiving the drug for only short term and in low doses ${ }^{18,19}$ as well as in longterm sedation of critically ill patients. ${ }^{20}$ The most common risk group for the development of PRIS are young, critically ill patients after neurosurgery on vasopressors with application of propofol with a mean dose of $\geq 4-5 \mathrm{mg} / \mathrm{kg} / \mathrm{h}$ over more than 48-hour duration. ${ }^{20}$

Midazolam, the other first-line sedative agent in neurocritical patients, is preferred over propofol in hemodynamically unstable patients. ${ }^{12,13}$ Midazolam offers a better hemodynamic profile than propofol with similar effects on neurophysiological parameters and metabolism markers. ${ }^{12,13}$ Its high liposolubility makes it more susceptible to tissue accumulation, confounding neurological examinations or hindering weaning from mechanical ventilation. Given the known anticonvulsant properties of midazolam, its association with spreading depolarization clusters is striking and should be confirmed, as well as the physiopathological mechanism behind it. ${ }^{21}$ During continuous infusion, tachyphylaxis may necessitate progressively higher doses. ${ }^{22}$ At doses used in status epilepticus stage 2-3, it causes respiratory depression, requiring intubation for the duration of therapy. ${ }^{22}$ Other benzodiazepines such as lorazepam are sometimes used, but their longer half-lives discourage their use in neurocritical patients. ${ }^{5}$

A promising alternative is dexmedetomidine, a central $\alpha_{2}$ adrenergic agonist with a short half-life that does not produce respiratory depression. Neuroprotective properties have been attributed to it, and one publication found an improvement in cognitive scores when compared with propofol. ${ }^{23,24}$ However, no evidence has been found for the purported lessened disturbance of neurophysiological parameters or improved outcome using dexmedetomidine under standard doses. ${ }^{9,25}$ No brain ischemia is to be expected under this drug if mean arterial pressure (MAP) is maintained. ${ }^{11,26}$ Dexmedetomidine does not have to be interrupted for neurological examinations. Its effects on neurophysiological parameters were not significantly different compared with other agents in discontinuous infusion. ${ }^{9}$ Low $(0.01-0.2 \mu \mathrm{g} / \mathrm{kg} / \mathrm{h})$ but not standard doses $(>0.2 \mu \mathrm{g} / \mathrm{kg} / \mathrm{h})$ of this drug were associated with a favorable outcome in comparison with propofol and midazolam. ${ }^{25}$ Main side effects of dexmedetomidine are hemodynamic changes (hypotension, bradycardia), but significant changes in cerebral blood supply can be prevented by maintaining MAP at baseline levels with vasopressors. ${ }^{11,25,26}$

Another alternative is ketamine, an $\mathrm{N}$-methyl-d-aspartate receptor antagonist that causes neither respiratory depression nor hemodynamic alterations. It is being recently reintroduced into the NICU for its short and rapid sedative effects. After several studies ruling out an increased risk of ICP elevation, including a meta-analysis, ketamine has regained increasing interest due to its influence on neural synapses. ${ }^{27}$ The encouraging results of its effects on spreading depolarization frequency, supported by in vitro studies, call for larger, confirmatory studies investigating its potential prognostic improvement in the neurocritical patient using analyses adjusted for prognostic factors. ${ }^{21,28}$ Ketamine can be used to reduce spreading depolarization frequency, though no concomitant improvement in prognosis has been proven. ${ }^{21}$

Barbiturates, due to their many side effects, are currently restricted to the treatment of refractory intracranial hypertension ${ }^{15}$ and status epilepticus. ${ }^{29}$ Finally, a single study evaluated low-dose thiopental against propofol. ${ }^{30}$ Due to the scarcity of data (patient characteristics, methods of measuring variables, protocol details, co-interventions), an unexplained age inclusion criterion (15-45 years), and grammatical and syntactic deficiencies that impaired understanding, its results were deemed as unreliable.

Opioids are commonly used in the ICU for analgesia, and the accumulation of opioid agents may cause respiratory depression, thus leading to prolongation of time to weaning and extubation. ${ }^{31}$ Studies have found the most frequently used opioids to be fentanyl (30-35\%), morphine (15-33\%), and sufentanil (25-40\%), the specific proportions of which differ in the results of different surveys, with remifentanil being less commonly used (10\%). ${ }^{32}$ However, the use of opioids may not be ideal in mechanically ventilated critically ill patients. Remifentanil has a highly predictable onset and offset effect (neurological evaluation), a terminal half-life of approximately 10 to 20 minutes, and a context-sensitive 
half-life of 3 to 4 minutes. The unique pharmacokinetics of remifentanil may lead to reductions in time to weaning and extubation and, accordingly, may be associated with reductions in mechanical ventilation time, length of ICU stay (ICU-LOS), and costs. ${ }^{31}$

In line with previous findings, no intravenous sedative agent has shown superiority over others, a prognostic improvement, or a decrease in mortality ${ }^{33}$ The results of our review highlight the need for studies designed to evaluate superiority and potential improvements in outcome.

\section{Volatile Anesthetics}

Inhalation anesthetic agents have in the past decade become available in the NICU through the use of a miniature vaporizer, the Anesthetic Conserving Device, AnaConDa (Sedana Medical $A B$, Sweden), which can be connected to any regular ICU ventilator. ${ }^{34}$ Their use has yielded some promising results in the general ICU, ${ }^{35,36}$ and some animal and invitro studies ${ }^{37,38}$ suggest that volatile anesthetic agents might have neuroprotective properties. The increase in cerebral blood flow (CBF) and reduction in cerebral metabolic rate make them an attractive alternative for patients with ABI. However, it remains unclear whether inhaled anesthetics are safe in these patients.

The available studies with isoflurane ${ }^{39,40}$ support the feasibility of using it as a sedative agent in the NICU. ICP remained stable, and the isoflurane-induced CBF redistribution could be particularly beneficial in cases of subarachnoid hemorrhage, as there is a high risk of CBF alterations (vasospasm) that worsen prognosis.

The studies with sevoflurane $e^{41-43}$ also support its potential use in the NICU, despite some discrepancies in cerebral perfusion pressure (CPP) that could be explained by differences in patient management protocols.

There is thus evidence for a lack of increase in ICP under volatile agents in most patients. Nevertheless, given the small sample sizes and short time under these drugs, it seems wise to restrict their use as sedatives to neurocritical patients with preserved CBF autoregulation (assessable with near-infrared spectroscopy), ${ }^{44}$ who can accept moderate increases in CBF without ICP elevation.

\section{Monitoring of Sedation in the Neurointensive Care Unit}

Conventional validated sedation scoring tools for critical care, such as the Richmond Agitation Sedation Scale and the Sedation-Agitation Scale, may be reasonable to use in patients with ABI. ${ }^{45}$ However, in deeply sedated patients or in those treated with neuromuscular blocking agents, the role of electroencephalogram (EEG) to monitor sedation has been a topic of clinical investigation. Simplified EEG tools providing quantitative bispectral index (BIS) monitoring showed that BIS values significantly correlated with Richmond Agitation Sedation Scale and Sedation-Agitation Scale scores in patients with ABI ${ }^{46}$ In another study, the BIS reliably assessed sedation levels during continuous propofol infusion in patients with TBI. ${ }^{47}$ Use of BIS in the NICU was limited by the reliability of these techniques (muscle artefacts, shivering) in the particular environment of the ICU. Also, the BIS was initially developed for monitoring the depth of general anesthesia in patients without brain pathology. ABI may influence the BIS algorithm because of EEG changes related to the pathology itself rather than to the sedative state. Whether new EEG techniques will allow better sedation monitoring in the NICU needs further investigation.

\section{Neurointensive Care Unit Sedation Practice}

Excluding sedation, most NICU therapeutic measures have been studied hitherto only in low-level evidence trials. Some have provided preliminary evidence to identify the practices that potentially improve prognosis, to be investigated in studies with greater scientific rigor. ${ }^{48}$ For example, tracheostomy has been associated with faster weaning from mechanical ventilation and reduction in $\mathrm{LOS}^{49}$ Pressure in the ICU to minimize sedation depth and duration to decrease delirium and cognitive impairment has sparked trials of similar protocols in the NICU. ${ }^{50}$ However, until data on neurophysiological and outcome variables are provided, no solid conclusions on the applicability of such protocols can be extracted. Deep sedation was only involved in one publication, ${ }^{51}$ without adjustment for prognostic factors or other studies confirming their results. There is currently insufficient evidence supporting its use over moderate or mild sedation.

The use of sedative agents for therapeutic effects other than sedation has also been studied. Sedation remains a nonsuperior therapeutic option in episodes of compromised $\mathrm{PbtO}_{2}$, which have been associated with increased mortality and worse prognosis. ${ }^{52-54}$ It is likely that specific subgroups of patients benefit more than others from specific interventions. Future investigations defining which patients benefit most from sedation will enable its use to correct $\mathrm{PbtO}_{2}$ reductions in a more targeted manner. The search for tools to differentiate the effect of sedatives from neurological damage is highly desirable in clinical decision-making. So far, it has been headed toward reliable scales to evaluate the patient's state of consciousness, ${ }^{45}$ though inherently subjective, and instruments that provide objective data, such as the BIS. ${ }^{55}$ The possibility of using conventional EEG to assess consciousness in sedated neurocritical patients, and isolate the effect of sedation as a confounder, has been introduced ${ }^{56}$ Note that the international 10-20 electrode placement system was used. Neurocritical patients will frequently have traumatic or surgical wounds or monitoring catheters, preventing the application of so many scalp EEG electrodes. A system with fewer electrodes has been successfully used in patients with $\mathrm{ABI}, 1,57$ but only for EEG seizure detection. There is yet no evidence that a conventional five-electrode EEG allows consciousness assessment in the neurocritical patient.

\section{Neurological Wake-Up Test}

Repeated neurological examinations aimed at detecting new neurological deficits are still the gold standard for neurocritical patient monitoring. ${ }^{58}$ In sedated patients with $\mathrm{ABI}$, they are called "NWT" and are performed after interruption of sedation (except with dexmedetomidine, which need not be discontinued). A recent narrative review discussed technical aspects of NWTs and their role in neurocritical care monitoring. 59 
In the ICU, a meta-analysis failed to confirm the previously suggested association of daily interruption of sedation with reduced duration of mechanical ventilation and LOS or with improved outcome. ${ }^{60}$ Thus, currently, daily interruption of sedation is not considered superior to protocol-guided sedation, but it is recommended in the absence of contraindications in all mechanically ventilated patients.,61 An ICU nursing-implemented protocol of daily interruption of sedation was evaluated, with inconclusive results. ${ }^{62}$ Note that such protocols do not include all the elements of the NWT and are not specifically aimed at detection of neurological worsening. Skoglund et al have provided most of the evidence currently available on NWT in the NICU. ${ }^{63-65}$ Their findings regarding ICP and the NWT-triggered sympathetic stress response are supported by two other studies. ${ }^{66,67}$ In all five studies, the stress response, together with co-interventions (vasopressor support), is likely to be responsible for elevated CPP in the context of an increased ICP.

The tendency toward metabolic distress in the failed-NWT group provides potential markers of NWT failure and neurological worsening, namely increased lactate-pyruvate ratio and lower glucose concentration in microdialysate. ${ }^{66}$ Notably, the NWT led to a change in the therapeutic approach of only 1 patient out of 20 participants. ${ }^{66}$ The findings of one study group suggest that NTWs may have prognostic value. ${ }^{67}$ Altogether, these data confirm that NWTs can be carried out safely in many, but not in all, neurocritical patients. ${ }^{63-67}$ Its benefits are yet unclear. This hinders the implementation of NWTs in many NICUs. ${ }^{68}$ Future studies should define which subgroup of patients might benefit from NWTs and in which the risks outweigh the potential benefits.

\section{Conclusion}

Whereas most studies consider intravenous sedatives, specifically propofol, midazolam, dexmedetomidine, and ketamine, widespread confounding factors (co-interventions, consecutive or simultaneous administration of sedatives, etc.) preclude superiority assessment. Inhaled sedative agents (isoflurane, sevoflurane), administered using the AnaConDa system, do not produce a clinically significant rise in ICP, but neither are a superior alternative to intravenous agents. There is no evidence that any particular sedative agent is superior or valid for all neurocritical patients or for all pathologies. Further studies are required to define which subgroup of neurocritical patients might benefit from NWT, and its potential prognostic value, as the possible ICP elevation during the NWT, may be harmful. Large-scale studies, designed to avoid confounding (especially regarding co-interventions) and evaluate superiority, are required.

\section{Conflict of Interest}

None declared.

\section{References}

1 Lindgren C, Nordh E, Naredi S, Olivecrona M. Frequency of non-convulsive seizures and non-convulsive status epilepticus in subarachnoid hemorrhage patients in need of controlled ventilation and sedation. Neurocrit Care 2012;17(3):367-373
2 Miller MA, Govindan S, Watson SR, Hyzy RC, Iwashyna TJ. $\mathrm{ABCDE}$, but in that order? A cross-sectional survey of Michigan intensive care unit sedation, delirium, and early mobility practices. Ann Am Thorac Soc 2015;12(7):1066-1071

3 Oldham M, Pisani MA. Sedation in critically ill patients. Crit Care Clin 2015;31(3):563-587

4 Klompas M, Li L, Szumita P, Kleinman K, Murphy MV; CDC Prevention Epicenters Program. Associations between different sedatives and ventilator-associated events, length of stay, and mortality in patients who were mechanically ventilated. Chest 2016;149(6):1373-1379

5 Devlin JW, Skrobik Y, Gélinas C, et al. Clinical practice guidelines for the prevention and management of pain, agitation/ sedation, delirium, immobility, and sleep disruption in adult patients in the ICU. Crit Care Med 2018;46(9):e825-e873

6 Beretta L, De Vitis A, Grandi E. Sedation in neurocritical patients: is it useful? Minerva Anestesiol 2011;77(8):828-834

7 Hukkelhoven CWPM, Steyerberg EW, Farace E, Habbema JDF, Marshall LF, Maas AIR. Regional differences in patient characteristics, case management, and outcomes in traumatic brain injury: experience from the tirilazad trials. J Neurosurg 2002;97(3):549-557

8 Teitelbaum JS, Ayoub O, Skrobik Y. A critical appraisal of sedation, analgesia and delirium in neurocritical care. Can J Neurol Sci 2011;38(6):815-825

9 James ML, Olson DM, Graffagnino C. A pilot study of cerebral and haemodynamic physiological changes during sedation with dexmedetomidine or propofol in patients with acute brain injury. Anaesth Intensive Care 2012;40(6):949-957

10 Tanguy M, Seguin P, Laviolle B, Bleichner JP, Morandi X, Malledant Y. Cerebral microdialysis effects of propofol versus midazolam in severe traumatic brain injury. J Neurotrauma 2012;29(6):1105-1110

11 Pajoumand M, Kufera JA, Bonds BW, et al. Dexmedetomidine as an adjunct for sedation in patients with traumatic brain injury. J Trauma Acute Care Surg 2016;81(2):345-351

12 Hertle DN, Santos E, Hagenston AM, et al. Cerebral glucose metabolism and sedation in brain-injured patients: a microdialysis study. J Neurosurg Anesthesiol 2015;27(3):187-193

13 Hertle DN, Beynon C, Neumann JO, et al. Use of GABAergic sedatives after subarachnoid hemorrhage is associated with worse outcome-preliminary findings. J Clin Anesth 2016;35:118-122

14 Hutchens MP, Memtsoudis S, Sadovnikoff N. Propofol for sedation in neuro-intensive care. Neurocrit Care 2006;4(1):54-62

15 Carney N, Totten AM, O'Reilly C, et al. Guidelines for the management of severe traumatic brain injury, fourth edition. Neurosurgery 2017;80:6-15

16 Parke TJ, Stevens JE, Rice AS, et al. Metabolic acidosis and fatal myocardial failure after propofol infusion in children: five case reports. BMJ 1992;305(6854):613-616

17 Marinella MA. Lactic acidosis associated with propofol. Chest 1996;109(1):292

18 Merz TM, Regli B, Rothen HU, Felleiter P. Propofol infusion syndrome-a fatal case at a low infusion rate. Anesth Analg 2006;103(4):1050

19 Kneiseler G, Bachmann HS, Bechmann LP, et al. A rare case of propofol-induced acute liver failure and literature review. Case Rep Gastroenterol 2010;4(1):57-65

20 Cremer OL, Moons KG, Bouman EA, Kruijswijk JE, de Smet AM, Kalkman CJ. Long-term propofol infusion and cardiac failure in adult head-injured patients. Lancet 2001;357(9250):117-118

21 Hertle DN, Dreier JP, Woitzik J, et al; Cooperative Study of Brain Injury Depolarizations (COSBID). Effect of analgesics and sedatives on the occurrence of spreading depolarizations accompanying acute brain injury. Brain 2012;135(Pt 8): 2390-2398

22 Zaccara G, Giannasi G, Oggioni R, Rosati E, Tramacere L, Palumbo P; convulsive status epilepticus study group of 
the uslcentro Toscana, Italy. Challenges in the treatment of convulsive status epilepticus. Seizure 2017;47:17-24

23 Jiang L, Hu M, Lu Y, Cao Y, Chang Y, Dai Z. The protective effects of dexmedetomidine on ischemic brain injury: A metaanalysis. J Clin Anesth 2017;40:25-32

24 Mirski MA, Lewin JJ III, Ledroux S, et al. Cognitive improvement during continuous sedation in critically ill, awake and responsive patients: the Acute Neurological ICU Sedation Trial (ANIST) Intensive Care Med 2010;36(9):1505-1513

25 Okazaki T, Hifumi T, Kawakita K, et al. Association between dexmedetomidine use and neurological outcomes in aneurysmal subarachnoid hemorrhage patients: a retrospective observational study. J Crit Care 2018;44:111-116

26 Wang X, Ji J, Fen L, Wang A. Effects of dexmedetomidine on cerebral blood flow in critically ill patients with or without traumatic brain injury: a prospective controlled trial. Brain Inj 2013;27(13-14):1617-1622

27 Himmelseher S, Durieux ME. Revising a dogma: ketamine for patients with neurological injury? Anesth Analg 2005; 101(2):524-534

28 Reinhart KM, Shuttleworth CW. Ketamine reduces deleterious consequences of spreading depolarizations. Exp Neurol 2018;305:121-128

29 Rossetti AO, Lowenstein DH. Management of refractory status epilepticus in adults: still more questions than answers. Lancet Neurol 2011;10(10):922-930

30 Yaghoobi S, Khezri MB, Alamouti AM. A pilot study of cerebral and hemodynamic changes during sedation with low dose of thiopental sodium or propofol in patients with acute brain injury. J Clin Diagn Res 2015;9(8):UC05-UC07

31 Zhu Y, Wang Y, Du B, Xi X. Could remifentanil reduce duration of mechanical ventilation in comparison with other opioids for mechanically ventilated patients? A systematic review and meta-analysis. Crit Care 2017;21(1):206

32 Payen JF, Chanques G, Mantz J, et al. Current practices in sedation and analgesia for mechanically ventilated critically ill patients: a prospective multicenter patient-based study. Anesthesiology 2007;106(4):687-695, quiz 891-892

33 Roberts DJ, Hall RI, Kramer AH, Robertson HL, Gallagher $\mathrm{CN}$, Zygun DA. Sedation for critically ill adults with severe traumatic brain injury: a systematic review of randomized controlled trials. Crit Care Med 2011;39(12):2743-2751

34 Belda JF, Soro M, Badenes R, et al. The predictive performance of a pharmacokinetic model for manually adjusted infusion of liquid sevofluorane for use with the AnestheticConserving Device (AnaConDa): a clinical study. Anesth Analg 2008;106(4):1207-1214

35 Soukup J, Schärff K, Kubosch K, Pohl C, Bomplitz M, Kompardt J. State of the art: sedation concepts with volatile anesthetics in critically Ill patients. J Crit Care 2009;24(4):535-544

36 Kim HY, Lee JE, Kim HY, Kim J. Volatile sedation in the intensive care unit: a systematic review and meta-analysis. Medicine (Baltimore) 2017;96(49):e8976

37 Kitano H, Kirsch JR, Hurn PD, Murphy SJ. Inhalational anesthetics as neuroprotectants or chemical preconditioning agents in ischemic brain. J Cereb Blood Flow Metab 2007; 27(6):1108-1128

38 Yang T, Sun Y, Zhang F. Anti-oxidative aspect of inhaled anesthetic gases against acute brain injury. Med Gas Res 2016;6(4):223-226

39 Bösel J, Purrucker JC, Nowak F, et al. Volatile isoflurane sedation in cerebrovascular intensive care patients using AnaConDa $\left({ }^{\circledR}\right)$ : effects on cerebral oxygenation, circulation, and pressure. Intensive Care Med 2012;38(12):1955-1964

40 Villa F, Iacca C, Molinari AF, et al. Inhalation versus endovenous sedation in subarachnoid hemorrhage patients: effects on regional cerebral blood flow. Crit Care Med 2012;40(10):2797-2804

41 Bisbal M, Arnal J-M, Passelac A, et al. Efficacy, safety and cost of sedation with sevoflurane in intensive care unit [article in French] Ann Fr Anesth Reanim 2011;30(4):335-341

42 Purrucker JC, Renzland J, Uhlmann L, et al. Volatile sedation with sevoflurane in intensive care patients with acute stroke or subarachnoid haemorrhage using AnaConDa®: an observational study. Br J Anaesth 2015;114(6):934-943

43 Badenes R, Bilotta F. Inhaled sedation in acute brain injury patients. Br J Anaesth 2016;116(6):883-884

44 Healy RJ, Zorrilla-Vaca A, Ziai W, et al. Glasgow coma scale score fluctuations are inversely associated with a NIRSbased Index of cerebral autoregulation in acutely comatose patients. J Neurosurg Anesthesiol 2018 May 18. doi:10.1097/ ANA.0000000000000513.

45 Riker RR, Fugate JE; Participants in the International Multidisciplinary Consensus Conference on Multimodality Monitoring. Clinical monitoring scales in acute brain injury: assessment of coma, pain, agitation, and delirium. Neurocrit Care 2014;21(Suppl 2):S27-S37

46 Deogaonkar A, Gupta R, DeGeorgia M, et al. Bispectral Index monitoring correlates with sedation scales in brain-injured patients. Crit Care Med 2004;32(12):2403-2406

47 Ogilvie MP, Pereira BM, Ryan ML, et al. Bispectral index to monitor propofol sedation in trauma patients. J Trauma 2011;71(5):1415-1421

48 Kurtz P, Fitts V, Sumer Z, et al. How does care differ for neurological patients admitted to a neurocritical care unit versus a general ICU? Neurocrit Care 2011;15(3):477-480

49 Lazaridis C, DeSantis SM, McLawhorn M, Krishna V. Liberation of neurosurgical patients from mechanical ventilation and tracheostomy in neurocritical care. J Crit Care 2012;27(4):417.e1-417.e8

50 Mahmoud L, Zullo AR, Thompson BB, Wendell LC. Outcomes of protocolised analgesia and sedation in a neurocritical care unit. Brain Inj 2018;32(7):941-947

51 Hou D, Liu B, Zhang J, Wang Q Zheng W. Evaluation of the efficacy and safety of short-course deep sedation therapy for the treatment of intracerebral hemorrhage after surgery: a non-randomized control study. Med Sci Monit 2016;22:2670-2678

52 Bohman L-E, Heuer G, Macyszyn L, et al. Medical management of compromised brain oxygen in patients with severe traumatic brain injury. Neurocrit Care 2011;14(3):361-369

53 Pascual JL, Georgoff P, Maloney-Wilensky E, et al. Reduced brain tissue oxygen in traumatic brain injury: are most commonly used interventions successful? J Trauma 2011;70(3):535-546

54 Rhodes JK, Chandrasekaran S, Andrews PJ. Early changes in brain oxygen tension may predict outcome following severe traumatic brain injury. Acta Neurochir Suppl (Wien) 2016;122:9-16

55 Yan K, Pang L, Gao H, et al. The influence of sedation level guided by bispectral index on therapeutic effects for patients with severe traumatic brain injury. World Neurosurg 2018;110:e671-e683

56 Claassen J, Velazquez A, Meyers E, et al. Bedside quantitative electroencephalography improves assessment of consciousness in comatose subarachnoid hemorrhage patients. Ann Neurol 2016;80(4):541-553

57 Olivecrona M, Zetterlund B, Rodling-Wahlström M, Naredi $\mathrm{S}$, Koskinen L-OD. Absence of electroencephalographic seizure activity in patients treated for head injury with an intracranial pressure-targeted therapy. J Neurosurg 2009; 110(2):300-305

58 Sharshar T, Citerio G, Andrews PJD, et al. Neurological examination of critically ill patients: a pragmatic approach. 
Report of an ESICM expert panel. Intensive Care Med 2014; 40(4):484-495

59 Marklund N. The neurological wake-up test-a role in neurocritical care monitoring of traumatic brain injury patients? Front Neurol 2017;8:540

60 Augustes R, Ho KM. Meta-analysis of randomised controlled trials on daily sedation interruption for critically ill adult patients. Anaesth Intensive Care 2011;39(3):401-409

61 Baron R, Binder A, Biniek R, et al; DAS-Taskforce 2015. Evidence and consensus based guideline for the management of delirium, analgesia, and sedation in intensive care medicine. Revision 2015 (DAS-Guideline 2015)-short version. Ger Med Sci 2015;13:Doc19

62 Anifantaki S, Prinianakis G, Vitsaksaki E, et al. Daily interruption of sedative infusions in an adult medical-surgical intensive care unit: randomized controlled trial. J Adv Nurs 2009;65(5):1054-1060

63 Skoglund K, Enblad P, Marklund N. Effects of the neurological wake-up test on intracranial pressure and cerebral perfusion pressure in brain-injured patients. Neurocrit Care 2009;11(2):135-142
64 Skoglund K, Enblad P, Hillered L, Marklund N. The neurological wake-up test increases stress hormone levels in patients with severe traumatic brain injury. Crit Care Med 2012;40(1):216-222

65 Skoglund K, Hillered L, Purins K, et al. The neurological wake-up test does not alter cerebral energy metabolism and oxygenation in patients with severe traumatic brain injury. Neurocrit Care 2014;20(3):413-426

66 Helbok R, Kurtz P, Schmidt MJ, et al. Effects of the neurological wake-up test on clinical examination, intracranial pressure, brain metabolism and brain tissue oxygenation in severely brain-injured patients. Crit Care 2012;16(6):R226

67 Esnault P, Montcriol A, D'Aranda E, et al. Early neurological wake-up test in intubated brain-injured patients: a long-term, single-centre experience. Aust Crit Care 2017;30(5):273-278

68 Herzer G, Mirth C, Illievich UM, Voelckel WG, Trimmel H. Analgosedation of adult patients with elevated intracranial pressure: survey of current clinical practice in Austria. Wien Klin Wochenschr 2018;130(1-2):45-53 